\title{
MATRICES WITH EIGENVECTORS IN A GIVEN SUBSPACE
}

\author{
GIORGIO OTTAVIANI AND BERND STURMFELS
}

(Communicated by Harm Derksen)

Abstract. The Kalman variety of a linear subspace in a vector space consists of all endomorphisms that possess an eigenvector in that subspace. We study the defining polynomials and basic geometric invariants of the Kalman variety.

\section{INTRODUCTION}

Let $L$ be a fixed $d$-dimensional linear subspace in the vector space $\mathbb{K}^{n}$, where $\mathbb{K}$ is an algebraically closed field. We are interested in the set $\mathcal{K}_{d, n}(L)$ of all $n \times n$ matrices $A=\left(a_{i j}\right)$ with entries in $\mathbb{K}$ that have a non-zero eigenvector in $L$. This set is an algebraic variety, and our aim is to study the defining equations and geometric properties of $\mathcal{K}_{d, n}(L)$. To this end, we write $L=\left\{x \in \mathbb{K}^{n}: C \cdot x=0\right\}$, where $C$ is a fixed $(n-d) \times n$-matrix of rank $n-d$, and we form the Kalman matrix (also called the observability matrix)

$$
\left(\begin{array}{c}
C \\
C A \\
C A^{2} \\
\vdots \\
C A^{d}
\end{array}\right)
$$

The Kalman matrix (1.1) has $n$ columns and $(d+1)(n-d)$ rows. The rows are grouped into $d+1$ blocks of $n-d$ rows, with the $i$ th block being the $(n-d) \times n$ matrix $C A^{i-1}$. It is known that $\mathcal{K}_{d, n}(L)$ consists of all matrices $A$ such that the $n$ columns of the Kalman matrix (1.1) are linearly dependent. Equivalently, we have

Proposition 1.1. The set $\mathcal{K}_{d, n}(L)$ is an algebraic variety in the matrix space $\mathbb{K}^{n \times n}$. It is the common zero set of the $n \times n$-minors of the Kalman matrix (1.1).

Proposition 1.1 is a variant of a classical result in control theory, known as Kalman's Observability Condition. A closely related formulation is the PBH Criterion (see [6] and 9]). A self-contained algebraic proof of Proposition 1.1 appears in [17, Theorem 2.1]. We generalize this in Theorem 4.5], which implies Proposition 1.1 as its special case $s=1$. Since the minors of (1.1) are homogeneous polynomials, we may regard the Kalman variety $\mathcal{K}_{d, n}(L)$ as a subvariety in the projective space $\mathbb{P}^{n^{2}-1}=\mathbb{P}\left(\mathbb{K}^{n \times n}\right)$. The basic invariants of this projective variety are as follows:

Received by the editors December 7, 2010 and, in revised form, May 23, 2011 and August 19, 2011.

2010 Mathematics Subject Classification. Primary 15A18; Secondary 13P25, 14N15, 93B25.

Key words and phrases. Eigenvectors, Kalman's observability condition, determinantal varieties, Gröbner bases, Hilbert series, vector bundles, Chern classes, resolution of singularities. 
Proposition 1.2. The Kalman variety $\mathcal{K}_{d, n}(L)$ is irreducible, and it has codimension $n-d$ and degree $\left(\begin{array}{c}n \\ d-1\end{array}\right)$ in $\mathbb{P}^{n^{2}-1}$.

The rank condition on the Kalman matrix is well known in control theory as a criterion for observability of linear systems. One of the earliest references to this matrix is Corollary 5.5 in Kalman's article [10]. Remarkably, no algebraist seems to have followed up and conducted the kind of study we offer here. The one possible exception we found is the work of Osetinskil et al. [13, who examined maximal minors of the Kalman matrix from the perspective of invariant theory and combinatorics.

The irreducibility and dimension part of Proposition 1.2 was proved in the 1983 $\mathrm{PhD}$ dissertation of Uwe Helmke, where he used it to compute the homotopy groups of the space of observable pairs $(A, C)$. The desingularization technique of our Section 4 is close to [7, where the approach was to look at the moment map in the setting of symplectic geometry. A point of view related to ours is also found in [3].

To express the equations of the Kalman variety in their simplest form, we shall assume, without loss of generality, that the given subspace $L \simeq \mathbb{K}^{d}$ is spanned by the first $d$ standard basis vectors $e_{1}, \ldots, e_{d}$, and we take $C=\left(\mathbf{0}_{(n-d) \times d} \mid \mathbf{I}_{(n-d) \times(n-d)}\right)$, a zero matrix prepended to an identity matrix. Given any $M \in \mathbb{K}^{n \times n}$ we write $[M]$ for the $(n-d) \times d$-matrix obtained from $M$ by taking the last $n-d$ rows and the first $d$ columns. With this notation, we define the small Kalman matrix to be

$$
\left(\begin{array}{c}
{[A]} \\
{\left[A^{2}\right]} \\
\vdots \\
{\left[A^{d}\right]}
\end{array}\right)
$$

This small Kalman matrix (1.2) has format $d(n-d) \times d$. The $d \times d$-minors of (1.2) and the $n \times n$-minors of (1.1) generate the same ideal $I_{d, n}$ in the polynomial ring $\mathbb{K}[A]=\mathbb{K}\left[a_{11}, a_{12}, \ldots, a_{n n}\right]$. The variety of $I_{d, n}$ is now simply denoted by $\mathcal{K}_{d, n}$, which is $\mathcal{K}_{d, n}(L)$ for $L=\mathbb{K}\left\{e_{1}, \ldots, e_{d}\right\}$. We conjecture that $I_{d, n}$ is a prime ideal.

Example 1.3. Let $n=4, d=2, L=\mathbb{K}\left\{e_{1}, e_{2}\right\}$. The Kalman variety $\mathcal{K}_{2,4}$ is the subset of $\mathbb{P}^{15}$ consisting of $4 \times 4$-matrices $A$ that have an eigenvector with the last two coordinates zero. It has codimension 2 and degree 4 . The small Kalman matrix is

$$
\left(\begin{array}{cc}
a_{31} & a_{32} \\
a_{41} & a_{42} \\
a_{11} a_{31}+a_{21} a_{32}+a_{31} a_{33}+a_{34} a_{41} & a_{12} a_{31}+a_{22} a_{32}+a_{32} a_{33}+a_{34} a_{42} \\
a_{11} a_{41}+a_{21} a_{42}+a_{31} a_{43}+a_{41} a_{44} & a_{12} a_{41}+a_{22} a_{42}+a_{32} a_{43}+a_{42} a_{44}
\end{array}\right) .
$$

The six $2 \times 2$-minors of this matrix (one quadric, four cubics and one quartic) generate the ideal $I_{2,4}$ but not minimally. To generate $I_{2,4}$, the quadric and certain three of the four cubics suffice. We note that $I_{2,4}$ is the prime ideal of $\mathcal{K}_{2,4}$.

Example 1.4. Let $n=5$ and $d=3$. The Kalman variety $\mathcal{K}_{3,5}$ has codimension 2 and degree 10 . It consists of $5 \times 5$-matrices $A$ that have an eigenvector with vanishing last three coordinates. The small Kalman matrix has size $6 \times 3$ and it equals

$$
\left(\begin{array}{c}
A_{21} \\
A_{21} A_{11}+A_{22} A_{21} \\
A_{21} A_{11}^{2}+A_{22} A_{21} A_{11}+A_{21} A_{12} A_{21}+A_{22}^{2} A_{21}
\end{array}\right),
$$


where we use the block decomposition

$$
A=\left(\begin{array}{ll}
A_{11} & A_{12} \\
A_{21} & A_{22}
\end{array}\right)
$$

with $A_{11}$ of size $3 \times 3, A_{12}$ of size $3 \times 2, A_{21}$ of size $2 \times 3$, and $A_{22}$ of size $2 \times 2$. The ideal $I_{3,5}$ is prime. It is minimally generated by eight of the twenty $3 \times 3$-minors of (1.3), namely, two quartics, two of the four quintics and four of the eight sextics.

We now introduce one further simplification in the representation of the Kalman variety $\mathcal{K}_{d, n}$. Since the condition that $A$ have an eigenvector in $L=\mathbb{K}\left\{e_{1}, \ldots, e_{d}\right\}$ does not depend on the entries in the last $n-d$ rows of $A$, we can set $A_{12}=A_{22}=0$ in the small Kalman matrix. This simplifies the $d \times d$-minors, but does not change the ideal $I_{d, n}$ they generate. It is instructive to check this in Examples 1.3 and 1.4.

We define the reduced Kalman matrix to be the $d(n-d) \times d$-matrix

$$
\left(\begin{array}{c}
A_{21} \\
A_{21} A_{11} \\
\vdots \\
A_{21} A_{11}^{d-1}
\end{array}\right)
$$

where $A_{11}$ is the upper left $d \times d$-block and $A_{21}$ is the lower left $(n-d) \times d$-block of $A$. Thus the reduced Kalman matrix is the small Kalman matrix with $A_{12}=A_{22}=0$.

In all practical computations with the ideal $I_{d, n}$ we always use the $d \times d$-minors of the reduced Kalman matrix as the set of generators. For instance, the ideal $I_{3,5}$ in Example 1.4 is generated by the maximal minors of the $6 \times 3$-matrix

$$
\left(\begin{array}{c}
A_{21} \\
A_{21} A_{11} \\
A_{21} A_{11}^{2}
\end{array}\right)
$$

where $A_{11}$ is a $2 \times 2$-matrix of variables and $A_{21}$ is a $3 \times 2$-matrix of variables.

This article is organized as follows. A self-contained proof of Proposition 1.2 is given in Section 2. In Section 3 we show that the generators of $I_{d, n}$ form a Gröbner basis when $d=2$. This seems to be no longer the case for $d \geq 3$. Section 4 concerns the singular locus of the Kalman variety $\mathcal{K}_{d, n}$, and this leads us to varieties defined by non-maximal minors of (1.5). In Section 5 we introduce vector bundle techniques for studying $\mathcal{K}_{d, n}$, and we compute the degrees of the singular locus.

Our results on the Kalman variety have the potential of being useful for a wide range of applications other than classical control theory and PDE. One example is the work of Tran [19] on statistical ranking. In her analysis of Saaty's analytical hierarchy process [14, 15, Tran examines the following question: Given a positive real $n \times n$-matrix $A$, under which condition does the dominant (Perron-Frobenius) eigenvector of $A$ fail to have distinct entries? The algebraic condition for the $i$ th and $j$ th coordinate of that eigenvector to be equal is precisely the Kalman variety of the hyperplane $L=\left\{x \in \mathbb{R}^{n}: x_{i}=x_{j}\right\}$. More generally, if $L$ is any linear space defined by equations of the form $x_{i}=x_{j}$, then the Kalman variety of $L$ arises in the stratification of the matrix space $\mathbb{R}^{n \times n}$ that represents distinct outcomes in [15]. 


\section{Dimension AND DEGREe}

In this section we present a proof of Proposition 1.2, We begin with an example.

Example 2.1. Consider the case $d=1$ when $L=\operatorname{ker}(C)$ is spanned by a single vector $v \in \mathbb{K}^{n} \backslash\{0\}$. Here, the Kalman variety $\mathcal{K}_{1, n}(L)$ is a linear space of codimension $n-1$. Its ideal $I_{1, n}(L)$ is generated by the $2 \times 2$-minors of the $n \times 2$-matrix $(v, A v)$. That same ideal of linear forms in $\mathbb{K}[A]$ is generated, highly redundantly, by the $\left(\begin{array}{c}2 n-2 \\ n\end{array}\right)$ maximal minors of the Kalman matrix $\left(\begin{array}{c}C \\ C A\end{array}\right)$, which has format $(2 n-2) \times n$. Clearly, it suffices to take only those $n-1$ maximal minors that involve the first $n-1$ rows and are hence linear in $A$. If we take $v=e_{1}$, then both descriptions yield the ideal $I_{1, n}=\left\langle a_{21}, a_{31}, \ldots, a_{n 1}\right\rangle$ whose generators are the entries of the reduced Kalman matrix $[A]$ of format $(n-1) \times 1$.

Proof of Proposition 1.2. We regard vectors $v \in \mathbb{K}^{n} \backslash\{0\}$ as points in the projective space $\mathbb{P}^{n-1}$, and we regard non-zero $n \times n$-matrices as points in $\mathbb{P}^{n^{2}-1}$. The product of these two projective spaces, $X=\mathbb{P}^{n^{2}-1} \times \mathbb{P}^{n-1}$, has the two projections

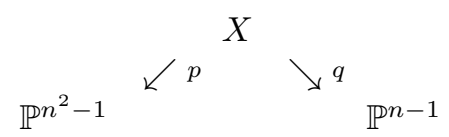

We fix the incidence variety $\mathcal{I}:=\left\{(A, v) \in \mathbb{P}^{n^{2}-1} \times \mathbb{P}^{n-1} \mid v\right.$ is a eigenvector of $\left.A\right\}$. The projection of $\mathcal{I}$ to the second factor

$$
\begin{array}{ccc}
\mathcal{I} & \stackrel{q}{\longrightarrow} & \mathbb{P}^{n-1} \\
(A, v) & \mapsto & v
\end{array}
$$

is surjective, and every fiber is a linear subspace of codimension $n-1$ in $\mathbb{P}^{n^{2}-1}$. To be precise, the fiber over $v$ is the linear space $\mathcal{K}_{1, n}(\mathbb{K} v)$ seen in Example 2.1.

The projection of the incidence variety $\mathcal{I}$ to the first factor

$$
\begin{array}{ccc}
\mathcal{I} & \stackrel{p}{\longrightarrow} & \mathbb{P}^{n^{2}-1} \\
(A, v) & \mapsto & A
\end{array}
$$

is surjective and the general fiber is finite. It consists of the $n$ eigenvectors of $A$. These properties imply that $\mathcal{I}$ is irreducible and has codimension $n-1$ in $X$.

The Kalman variety has the following description in terms of the diagram (2.1):

$$
\mathcal{K}_{d, n}(L)=p_{*}\left(\mathcal{I} \cap q^{*}(\mathbb{P}(L))\right) .
$$

The $(d-1)$-dimensional subspace $\mathbb{P}(L)$ of $\mathbb{P}^{n-1}$ specifies the following diagram:

$$
\mathcal{K}_{d, n}(L) \swarrow^{p} \searrow^{\mathcal{I} \cap q^{*}(\mathbb{P}(L))} \mathbb{P}(L)
$$

As before, each fiber of the map $q$ in (2.3) is a linear space of codimension $n-1$ in $\mathbb{P}^{n^{2}-1}$. This implies that $\mathcal{I} \cap q^{*}(\mathbb{P}(L))$ is irreducible and its dimension equals $\operatorname{dim}(\mathbb{P}(L))+\operatorname{dim}\left(\mathbb{P}^{n^{2}-1}\right)-(n-1)=(d-1)+n^{2}-1-(n-1)=n^{2}-1-(n-d)$.

Since the general fiber of the surjection $p$ is finite, the Kalman variety $\mathcal{K}_{d, n}(L)$ is irreducible of the same dimension. Hence $\mathcal{K}_{d, n}(L)$ has codimension $n-d$ in $\mathbb{P}^{n^{2}-1}$. 
We next derive the degree of $\mathcal{K}_{d, n}(L)$. Consider the prime ideal generated by the $2 \times 2$-minors of a $2 \times n$-matrix $(u, v)$. This ideal lives in an $\mathbb{N}^{2}$-graded polynomial ring $\mathbb{K}[u, v]$ in $2 n$ unknowns. Its multidegree, in the sense of [12, $\S 8.5]$, is equal to

$$
\mathcal{C}\left(t_{1}, t_{2}\right)=t_{1}^{n-1}+t_{1}^{n-2} t_{2}+t_{1}^{n-3} t_{2}^{2}+\cdots+t_{1} t_{2}^{n-2}+t_{2}^{n-1} .
$$

This formula is the case $k=2$ and $\ell=n$ of Exercise 15.5(b) in [12, page 308].

The prime ideal of the incidence variety $\mathcal{I} \subset \mathbb{P}^{n^{2}-1} \times \mathbb{P}^{n-1}$ lives in the $\mathbb{N}^{2}$-graded polynomial ring $\mathbb{K}[A, v]$ in $n^{2}+n$ unknowns, namely the entries of $A$ and of $v$. This prime ideal is generated by the $2 \times 2$-minors of the matrix $(A v, v)$, so it is obtained from the ideal in the previous paragraph by the bilinear substitution $u \mapsto A v$. This implies that the multidegree of $\mathcal{I}$ is obtained from (2.4) by the substitution $t_{1} \mapsto t_{1}+t_{2}$. Hence the multidegree of $\mathcal{I}$ is the bivariate polynomial

$$
\mathcal{C}\left(t_{1}+t_{2}, t_{2}\right)=\sum_{d=1}^{n}\left(t_{1}+t_{2}\right)^{n-d} t_{2}^{d-1}=\sum_{d=1}^{n}\left(\begin{array}{c}
n \\
d-1
\end{array}\right) t_{1}^{n-d} t_{2}^{d-1} .
$$

In geometric language, the bidegree (2.5) is the equivariant cohomology class of the incidence variety $\mathcal{I}$ with respect to the natural $\left(\mathbb{K}^{*}\right)^{2}$-action on $\mathbb{P}^{n^{2}-1} \times \mathbb{P}^{n-1}$.

The classical degree of a projective variety is the number of intersection points with a general linear subspace of complementary dimension. Likewise, each coefficient of the bidegree (2.5) is the number of points in the intersection of $\mathcal{I}$ with an $(n-1)$-dimensional variety $\mathbb{P}(K) \times \mathbb{P}(L)$, where $\mathbb{P}(K)$ and $\mathbb{P}(L)$ are general linear subspaces of $\mathbb{P}^{n^{2}-1}$ and $\mathbb{P}^{n-1}$. Specifically, if we fix $\mathbb{P}(L)$ of dimension $d-1$, then this number equals $\left(\begin{array}{c}n \\ d-1\end{array}\right)$. Equivalently, the number of points in the intersection of $\mathcal{K}_{d, n}(L)$ with a general $(n-d)$-dimensional linear subspace $\mathbb{P}(K)$ of $\mathbb{P}^{n^{2}-1}$ is equal to $\left(\begin{array}{c}n \\ d-1\end{array}\right)$. Therefore, $\operatorname{degree}\left(\mathcal{K}_{d, n}(L)\right)=\left(\begin{array}{c}n \\ d-1\end{array}\right)$, and the proof is complete.

Example 2.2. Consider the special case $d=n-1$ when $L$ is the hyperplane in $\mathbb{K}^{n}$ perpendicular to a non-zero row vector $C$. Then (1.1) is the $n \times n$-matrix whose $i$-th row vector is $C A^{i-1}$. The Kalman variety $\mathcal{K}_{n-1, n}$ is the hypersurface in $\mathbb{P}^{n^{2}-1}$ of degree $\left(\begin{array}{l}n \\ 2\end{array}\right)$ defined by the determinant of that matrix. Likewise, the reduced Kalman matrix (1.5) is the $(n-1) \times(n-1)$-matrix whose $i$-th row vector equals $A_{21} A_{11}^{i-1}$. Its determinant is that same generator of the principal ideal $I_{n-1, n}$.

\section{Combinatorics FOR $d=2$}

In this section we fix $d=2$. We present a study of the ideal $I_{2, n}$ from the perspective of combinatorial commutative algebra [12. The reduced Kalman matrix is

$$
\left(\begin{array}{c}
A_{21} \\
A_{21} A_{11}
\end{array}\right)
$$

where $A_{11}$ is the upper left $2 \times 2$-block and $A_{21}$ is the lower left $(n-2) \times 2$-block of the $n \times n$-matrix $A=\left(a_{i j}\right)$. The Kalman variety $\mathcal{K}_{2, n}$ has codimension $n-2$, and the Kalman ideal $I_{2, n}$ is generated by the $2 \times 2$-minors of the $(2 n-4) \times 2$-matrix (3.1).

This construction has the following geometric interpretation. Consider the Segre variety $\mathbb{P}^{1} \times \mathbb{P}^{n-1} \subset \mathbb{P}^{2 n-1}$ that is cut out by the $2 \times 2$-minors of the $n \times 2$-matrix $\left(\begin{array}{l}A_{11} \\ A_{21}\end{array}\right)$. Take the cone over that Segre variety from the point $\left(\begin{array}{c}\mathbf{I}_{2 \times 2} \\ \mathbf{o}_{n-2 \times 2}\end{array}\right)$. That cone is a subvariety of codimension $n-2$ and degree $n$ in $\mathbb{P}^{2 n-1}$ defined by the $2 \times 2$-minors of (3.1). We conclude the following geometric description of our variety: 
Remark 3.1. The Kalman variety $\mathcal{K}_{2, n} \subset \mathbb{P}^{n^{2}-1}$ is the cone with base $\mathbb{P}^{n^{2}-2 n}$ over a general linear projection of the Segre variety $\mathbb{P}^{1} \times \mathbb{P}^{n-1}$ from $\mathbb{P}^{2 n-1}$ into $\mathbb{P}^{2 n-2}$.

Our goal in this section is to prove the following result about $\mathcal{K}_{2, n}$ and its ideal.

Theorem 3.2. The ideal $I_{2, n}$ is prime. Its minimal generators are the $\left(\begin{array}{c}n-2 \\ 2\end{array}\right)$ quadrics

$$
\underline{a_{i 1} a_{j 2}}-a_{i 2} a_{j 1}=\left|\begin{array}{cc}
a_{i 1} & a_{i 2} \\
a_{j 1} & a_{j 2}
\end{array}\right|, \quad \text { where } 3 \leq i<j \leq n,
$$

and the $\left(\begin{array}{c}n-1 \\ 2\end{array}\right)$ cubics

$$
\begin{aligned}
& \frac{a_{11} a_{i 2} a_{j 1}}{-}-a_{12} a_{i 1} a_{j 1}+a_{21} a_{i 2} a_{j 2}-a_{22} a_{i 1} a_{j 2} \\
& =-\left|\begin{array}{cc}
a_{i 1} & a_{i 2} \\
a_{j 1} a_{11}+a_{j 2} a_{21} & a_{j 1} a_{12}+a_{j 2} a_{22}
\end{array}\right|, \quad \text { where } 3 \leq i \leq j \leq n .
\end{aligned}
$$

These form a Gröbner basis of $I_{2, n}$ with respect to the lexicographic term order. The Hilbert series of the quotient ring $\mathbb{K}[A] / I_{2, n}$ with the standard $\mathbb{N}$-grading equals

$$
\frac{n}{(1-z)^{n^{2}-n+2}}-\frac{n-1}{(1-z)^{n^{2}-n+1}}-\frac{1}{(1-z)^{n^{2}-2 n+4}}+\frac{1}{(1-z)^{n^{2}-2 n+3}} .
$$

Proof. The listed quadrics and cubics are among the $2 \times 2$-minors of the reduced Kalman matrix, so they lie in the ideal $I_{2, n}$. Their lexicographic leading terms are the underlined monomials. Let $M$ denote the radical ideal generated by these monomials. Then we have $M \subseteq \operatorname{in}_{\text {lex }}\left(I_{2, n}\right)$. Our goal is to show that equality holds.

The radical monomial ideal $M$ is the intersection of $n$ monomial primes, each having codimension $n-2$. The corresponding simplicial complex $\Delta$ has the facets

$$
\begin{aligned}
& \left\{a_{11}, a_{31}, a_{41}, \ldots, a_{n 1}\right\}, \\
& \left\{a_{32}, a_{42}, \ldots, a_{i 2}, a_{i 1}, a_{i+1,1}, \ldots a_{n 1}\right\}, \text { for } i=3,4, \ldots, n, \\
& \left\{a_{11}, a_{32}, a_{42}, \ldots, a_{n 2}\right\} .
\end{aligned}
$$

and

Here we only list variables that appear in the generators of $M$, not the $n^{2}-2 n+3$ variables $a_{i j}$ that do not appear. The simplicial complex that represents the variety of $M$ in $\mathbb{P}^{n^{2}-1}$ is obtained from (3.5) by taking the join with a simplex of dimension $n^{2}-2 n+2$. From the list in (3.5) we see that $M$ is an unmixed ideal of codimension $n-2$ and degree $n$. Recall that being unmixed means that $M$ is the intersection of prime ideals each generated by a set of variables $a_{i j}$ of the same cardinality.

Since $I_{2, n}$ has codimension $n-2$ and degree $n$, by Proposition 1.2, the initial monomial ideal $\operatorname{in}_{\text {lex }}\left(I_{2, n}\right)$ also has codimension $n-2$ and degree $n$. But this implies that $M=\operatorname{in}_{\text {lex }}\left(I_{2, n}\right)$. Indeed, if the unmixed radical ideal $M$ were strictly contained in $\operatorname{in}_{\text {lex }}\left(I_{2, n}\right)$, then the latter would have lower degree or higher codimension.

This proves that the quadrics in (3.2) and cubics in (3.3) form a Gröbner basis for $I_{2, n}$, and in particular they generate $I_{2, n}$. This generating set is minimal because the generators are $\mathbb{K}$-linearly independent and none of the cubics can be written as a $\mathbb{K}[A]$-linear combination of the quadrics. We have shown that $\operatorname{in}_{\text {lex }}\left(I_{2, n}\right)$ is a radical ideal, and this implies that $I_{2, n}$ is radical. Since the variety $\mathcal{K}_{2, n}$ defined by $I_{2, n}$ is irreducible, we can conclude that the ideal $I_{2, n}$ is prime.

Let $\Delta^{\prime}$ be the simplicial complex of dimension $n-2$ obtained from $\Delta$ by replacing the occurrence of the vertex $a_{11}$ in the last facet of (3.5) with a different vertex $a_{11}^{\prime}$. Then the given ordering of the $n$ facets represents a shelling of $\Delta^{\prime}$. In that shelling order, precisely one new vertex enters whenever a new facet gets attached. 
This implies that the Hilbert series of the Stanley-Reisner ring $\mathbb{K}\left[\Delta^{\prime}\right]$ is equal to $(1+(n-1) z) /(1-z)^{n-1}$. When we pass from $\Delta^{\prime}$ to $\Delta$, we identify $\left(a_{11}^{\prime}\right)^{i}$ with $a_{11}^{i}$ for each $i \leq 1$, and all other standard monomials remain unchanged. Hence we lose one monomial per positive degree, so we need to subtract $z /(1-z)$ from the previous Hilbert series. This implies that $\mathbb{K}[\Delta]=\mathbb{K}[A] / M$, regarded as a quotient of the polynomial ring in $n^{2}$ variables, has the Hilbert series

$$
\frac{1}{(1-z)^{n^{2}-2 n+3}}\left(\frac{1+(n-1) z}{(1-z)^{n-1}}-\frac{z}{1-z}\right) \text {. }
$$

This is also the Hilbert series of $\mathbb{K}[A] / I_{2, n}$. It equals the rational function (3.4).

From the Hilbert function formula (3.4) we can read the Hilbert polynomial:

Corollary 3.3. The Hilbert polynomial of the Kalman variety $\mathcal{K}_{2, n}$ in $\mathbb{P}^{n^{2}-1}$ equals

$$
n\left[\mathbb{P}^{n^{2}-n+1}\right]-(n-1)\left[\mathbb{P}^{n^{2}-n}\right]-\left[\mathbb{P}^{n^{2}-2 n+3}\right]+\left[\mathbb{P}^{n^{2}-2 n+2}\right],
$$

where $\left[\mathbb{P}^{r}\right]=\left(\begin{array}{c}t+r \\ r\end{array}\right)$ is the Hilbert polynomial of $r$-dimensional projective space $\mathbb{P}^{r}$.

Remark 3.4. The Gröbner basis of quadrics and cubics in Theorem 3.2 is not reduced since the last term $a_{22} a_{i 1} a_{j 2}$ of (3.3) for $i<j$ is divisible by the leading term of (3.2). Replacing that last term by $a_{22} a_{i 2} a_{j 1}$, we get the reduced Gröbner basis.

Theorem 3.2 does not extend to $d \geq 3$. In particular, the lexicographic initial ideal $\operatorname{in}_{\text {lex }}\left(I_{3,5}\right)$ is not radical. At present we do not even know whether $I_{3, n}$ is prime and we do not have a general formula for its Hilbert polynomial.

Example 3.5. The Kalman variety for $n=9$ and $d=3$ has codimension 6 and degree 36 in $\mathbb{P}^{26}$. Its K-polynomial (i.e. the numerator of its Hilbert series) equals

$$
\begin{gathered}
(1-z)^{6} \cdot\left(1+6 z+21 z^{2}+36 z^{3}-19 z^{4}-109 z^{5}+151 z^{6}+152 z^{7}-637 z^{8}\right. \\
\left.+896 z^{9}-792 z^{10}+495 z^{11}-220 z^{12}+66 z^{13}-12 z^{14}+z^{15}\right)
\end{gathered}
$$

and its Hilbert polynomial is found to be

$36\left[\mathbb{P}^{20}\right]-63\left[\mathbb{P}^{19}\right]+28\left[\mathbb{P}^{18}\right]-42\left[\mathbb{P}^{14}\right]+111\left[\mathbb{P}^{13}\right]-97\left[\mathbb{P}^{12}\right]+28\left[\mathbb{P}^{11}\right]+\left[\mathbb{P}^{8}\right]-3\left[\mathbb{P}^{7}\right]+3\left[\mathbb{P}^{6}\right]-\left[\mathbb{P}^{5}\right]$.

In particular, for $d \geq 3$ there is no formula as short and simple as (3.7).

The following result about the generators of $I_{3, n}$ appeared as a conjecture in the first version of this paper. A proof was announced by Steven Sam in May 2011.

Theorem 3.6 (Sam [16]). The ideal $I_{3, n}$ is minimally generated by polynomials of degree $3,4,5,6$. There are $\left(\begin{array}{c}n-3 \\ 3\end{array}\right)$ generators in degree 3 , there are $2\left(\begin{array}{c}n-2 \\ 3\end{array}\right)$ generators each in degree 4 and in degree 5 , and there are $\left(\begin{array}{c}n-1 \\ 3\end{array}\right)$ generators in degree 6 .

\section{Singularities AND their Resolution}

In this section we study the singular locus of the Kalman variety $\mathcal{K}_{d, n}=\mathcal{K}_{d, n}(L)$, and we prove that the map $p$ in (2.3) is a resolution of singularities. By definition, the singular locus $\operatorname{Sing}\left(\mathcal{K}_{d, n}\right)$ is the subvariety of $\mathcal{K}_{d, n}$ defined by the vanishing of the minors of size $n^{2}-n+d+1$ in the Jacobian matrix of the generators of $I_{d, n}$.

Returning to the setting of Section 2, we consider the restricted incidence variety

$$
\mathcal{I} \cap q^{*}(\mathbb{P}(L))=\left\{(A, v) \in \mathbb{P}^{n^{2}-1} \times \mathbb{P}(L) \mid v \text { is a eigenvector of } A\right\} .
$$

This is a smooth variety because the fibers of the second projection $q$ to $\mathbb{P}(L)$ are linear spaces of the same dimension. For $d=1$, the Kalman variety is a linear space, so it is smooth. For $d \geq 2$, the Kalman variety $\mathcal{K}_{d, n}$ does have singularities: 
Lemma 4.1. For $2 \leq d \leq n-1$ the singular locus of $\mathcal{K}_{d, n}$ is given by the matrices A such that there exists a subspace $L^{\prime} \subseteq L$ of dimension 2 which is $A$-invariant.

Proof. Consider a general pair $(A, v) \in \mathcal{I} \cap q^{*}(\mathbb{P}(L))$. Then $v$ is the only eigenvector of $A$ in $L$ (up to scalar multiples). Therefore $p^{-1}(p(A, v))=p^{-1}(A)=\{(A, v)\}$; that is, the general fiber of the map $p$ in (2.3) consists of only one point. This means that the morphism $p$ is birational. On the other hand, if $A$ has two linearly independent eigenvectors in $L$, then the fiber $p^{-1}(A)$ consists of two points.

A standard result in algebraic geometry, known as Zariski's Main Theorem, states that the inverse image of a normal point under a birational projective morphism is connected. Hence the point $A$ is not normal in $\mathcal{K}_{d, n}$ when $\left|p^{-1}(A)\right| \geq 2$. We infer that $\operatorname{Sing}\left(\mathcal{K}_{d, n}\right)$ contains the set described in the statement of Lemma 4.1 This containment must be an equality because being singular is a local property, and any two points $A, A^{\prime} \in \mathcal{K}_{d, n}$ in the complement of that set are locally isomorphic. The latter property holds because $(A, v)$ and $\left(A^{\prime}, v^{\prime}\right)$ are locally isomorphic points in $\mathcal{I} \cap q^{*}(\mathbb{P}(L))$ if $v$, resp. $v^{\prime}$, is the unique eigenvector of $A$ resp. $A^{\prime}$ in $L$.

Corollary 4.2. The restricted incidence variety $\mathcal{I} \cap q^{*}(\mathbb{P}(L))$ is the desingularization of the Kalman variety $\mathcal{K}_{d, n}$. The map $p$ in (2.3) is a resolution of singularities.

Proof. The variety defined by the $2 \times 2$-minors of the matrix $(v, A v)$ is smooth. If we impose the constraints $v \in L$, for a general linear subspace $L \subset \mathbb{K}^{n}$, then the intersection remains smooth, by Bertini's Theorem. That intersection is precisely our incidence variety $\mathcal{I} \cap q^{*}(\mathbb{P}(L))$. However, that variety is independent of $L$.

Example 4.3. If $d=2$, then $\operatorname{Sing}\left(\mathcal{K}_{2, n}\right)$ consists precisely of the matrices $A$ whose lower left $(n-2) \times 2$-block $A_{21}$ is zero. Hence $\operatorname{Sing}\left(\mathcal{K}_{2, n}\right)$ is a linear subspace of codimension $2 n-4$. It contains the $\mathbb{P}^{n^{2}-2 n}$ over which $\mathcal{K}_{2, n}$ is a cone by Remark 3.1 .

We extend our description of the singular locus of the Kalman variety by defining $\mathcal{K}_{s, d, n}=\left\{A \in \mathbb{P}^{n^{2}-1} \mid\right.$ there is an $A$-invariant subspace of dimension $\geq s$ in $\left.L\right\}$.

Note that $\mathcal{K}_{d, d, n}$ is a linear space of codimension $d(n-d)$, consisting of the matrices $A$ whose lower left $(n-d) \times d$-block $A_{21}$ is zero. We have the chain of inclusions $\mathcal{K}_{d, d, n} \subseteq \mathcal{K}_{d-1, d, n} \subseteq \ldots \subseteq \mathcal{K}_{1, d, n}=\mathcal{K}_{d, n}$. The following theorem extends Lemma 4.1. It concerns the iterated singularity structure of the Kalman variety.

Theorem 4.4. For $1 \leq s \leq d-1$, the variety $\mathcal{K}_{s+1, d, n}$ is precisely the singular locus of $\mathcal{K}_{s, d, n}$. The codimension of $\mathcal{K}_{s, d, n}$ in $\mathbb{P}^{n^{2}-1}$ is $s(n-d)$, and the codimension of $\mathcal{K}_{s, d, n}$ in $\mathcal{K}_{s-1, d, n}$ equals $n-d$. In particular, $\operatorname{codim}\left(\operatorname{Sing}\left(\mathcal{K}_{d, n}\right)\right)=n-d$.

Proof. We generalize the incidence variety used in the proof of Lemma 4.1, Let

$$
\mathcal{K} \mathcal{S}_{s, d, n}=\left\{\left(A, L^{\prime}\right) \in \mathbb{P}^{n^{2}-1} \times G r(s, L) \mid L^{\prime} \text { is } A \text {-invariant }\right\} .
$$

This is a smooth variety of dimension $n^{2}-1-s(n-d)$ because the second projection to the Grassmannian $\operatorname{Gr}(s, L)$ is surjective and its fibers are linear spaces of dimension $n^{2}-1-s(n-s)$. The first projection $p$ is bijective when restricted to $p^{-1}\left(\mathcal{K}_{s, d, n} \backslash \mathcal{K}_{s+1, d, n}\right)$ because a matrix $A \in \mathcal{K}_{s, d, n} \backslash \mathcal{K}_{s+1, d, n}$ determines uniquely its $A$-invariant subspace $L^{\prime}$. It follows that $\mathcal{K S}_{s, d, n}$ is the desingularization of $\mathcal{K}_{s, d, n}$.

A general matrix $A \in K_{s+1, d, n}$ has $s+1$ independent eigenvectors in $L$, so its fiber $p^{-1}(A)$ consists of $\left(\begin{array}{c}s+1 \\ s\end{array}\right)=s+1$ distinct points. Again by Zariski's Main 
Theorem, these are singular points of $\mathcal{K}_{s, d, n}$, so that $\operatorname{Sing}\left(\mathcal{K}_{s, d, n}\right)$ contains $\mathcal{K}_{s+1, d, n}$. However, this containment is an equality, by the argument used for Lemma 4.1 .

We next state a result about the defining equations of the iterated singular loci.

Theorem 4.5. The variety $\mathcal{K}_{s, d, n}$ is the zero set of the $(n-s+1) \times(n-s+1)$-minors of the Kalman matrix (1.1), or equivalently the zero set of the $(d-s+1) \times(d-s+1)$ minors of the reduced Kalman matrix (1.5). In particular, $\operatorname{Sing}\left(\mathcal{K}_{d, n}\right)$ consists of all matrices $A$ such that the reduced Kalman matrix has rank $\leq d-2$.

Proof. We must show that an $n \times n$-matrix $A$ has an $s$-dimensional $A$-invariant subspace $L^{\prime} \subseteq L=\operatorname{ker}(C)$ if and only if the Kalman matrix (1.1) has rank $\leq n-s$. We begin with the only-if direction. If $A$ has an $s$-dimensional invariant subspace $L^{\prime} \subseteq L$, then every $v \in L^{\prime}$ satisfies $A^{i} v \in L^{\prime}$, which implies $C A^{i} v=0$. Hence $L^{\prime}$ is contained in the kernel of the Kalman matrix (1.1), which must have rank $\leq n-s$. Conversely, we prove that the kernel of the Kalman matrix is $A$-invariant. We assume that $L=\mathbb{K}\left\{e_{1}, \ldots, e_{d}\right\}$. Any vector $v$ in the kernel of the Kalman matrix has the form $v=\left(\begin{array}{c}w \\ 0\end{array}\right)$ with $A_{21} A_{11}^{i} w=0$ and $i=0,1, \ldots, d-1$. Then $A v=\left(\begin{array}{c}A_{11} w \\ 0\end{array}\right)$. This vector is in the kernel of the Kalman matrix if $A_{21} A_{11}^{i}\left(A_{11} w\right)=A_{21} A_{11}^{i+1} w=0$ for $i=0,1, \ldots, d-1$. Since $A_{11}^{d} w$ is a linear combination of $w, A_{11} w, \ldots, A_{11}^{d-1} w$, because $A_{11}$ is a $d \times d$-matrix, these equalities are satisfied. This shows that the kernel of the Kalman matrix is $A$-invariant. Hence $A \in \mathcal{K}_{s, d, n}$.

A case of special interest is $s=d-1$. The variety $\mathcal{K}_{d-1, d, n}$ is cut out by the $2 \times 2$-minors of the reduced Kalman matrix (1.5). Experimental evidence suggests that some of the nice combinatorics seen in Section 3 generalizes to this case. In particular, the ideal generated by these $2 \times 2$-minors of (1.5) appears to be prime, and we conjecture that its initial ideal in the lexicographic term order is radical.

We close this section by stating a formula for the degrees of our iterated singular loci. It will be proved in the next section. Our proof technique involves results from representation theory that require the field $\mathbb{K}$ to have characteristic 0 (see [5]).

Theorem 4.6. Let char $(\mathbb{K})=0$. The degree of the subvariety $\mathcal{K}_{s, d, n}$ in $\mathbb{P}^{n^{2}-1}$ is the coefficient of $\left(x_{1} x_{2} \cdots x_{s}\right)^{d-s}$ in the expansion of the symmetric rational function

$$
\frac{\prod_{i=1}^{s}\left(1+x_{i}\right)^{n}}{\prod_{i, j=1}^{s}\left(1+\left(x_{i}-x_{j}\right)\right)}
$$

as a $\mathbb{Z}$-linear combination of Schur polynomials $s_{\lambda}\left(x_{1}, \ldots, x_{s}\right)$. In particular, for fixed $d>s>0$, the degree of the variety $\mathcal{K}_{s, d, n}$ is a polynomial in $n$ of the form

$$
\operatorname{deg}\left(\mathcal{K}_{s, d, n}\right)=\frac{\operatorname{deg} G r(s, L)}{[s(d-s)] !} \cdot n^{s(d-s)}+\cdots(\text { lower terms in } n),
$$

where $\operatorname{deg} \operatorname{Gr}(s, L)=\frac{1 ! 2 ! \ldots(s-1) ![s(d-s)] !}{(d-s) !(d-s+1) ! \ldots(d-1) !} \quad$ is the degree of the Grassmannian.

Example 4.7. Consider the Kalman variety $\mathcal{K}_{3,5}$ in Example 1.4, Its singular locus $\mathcal{K}_{2,3,5}$ is the codimension 4 variety defined by the $2 \times 2$-minors of the $6 \times 3$-matrix in (1.4). Here $s=2, d=3, n=5$, and the symmetric rational function (4.1) equals $\frac{\left(1+x_{1}\right)^{5}\left(1+x_{2}\right)^{5}}{\left(1-\left(x_{1}-x_{2}\right)^{2}\right)}=1+5\left(x_{1}+x_{2}\right)+\mathbf{1 2} x_{1} x_{2}+11\left(x_{1}^{2}+x_{1} x_{2}+x_{2}^{2}\right)+$ higher terms.

We see that $\mathcal{K}_{2,3,5} \subset \mathbb{P}^{14}$ has degree 12 . The lexicographic Gröbner basis for $\mathcal{K}_{2,3,5}$ consists of 3 quadrics, 9 cubics and 3 quartics, with square-free initial terms. 


\section{Kalman varieties Via Vector BUndles}

In this section we develop a geometric interpretation of the Kalman variety and its desingularization, in terms of the tangent bundle of a suitable Grassmannian. This works also for the varieties $\mathcal{K}_{s, d, n}$ introduced in the previous section. This includes $\mathcal{K}_{d, n}$ for $s=1$. We shall assume from now on that $\mathbb{K}$ has characteristic 0 .

Let ad $\mathbb{K}^{n}$ denote the vector space of $n \times n$-matrices with trace zero. We shall consider our varieties in the corresponding projective space $\mathbb{P}^{n^{2}-2}=\mathbb{P}\left(\right.$ ad $\left.\mathbb{K}^{n}\right)$. To be precise, we write $\tilde{\mathcal{K}}_{s, d, n}$ for the intersection of $\mathcal{K}_{s, d, n}$ with $\mathbb{P}^{n^{2}-2}$. This is a subvariety of codimension $s(n-d)$. We do not lose any information when passing from $\mathcal{K}_{s, d, n}$ to $\tilde{\mathcal{K}}_{s, d, n}$, because $\mathcal{K}_{s, d, n}$ is a cone with vertex at the identity matrix $\mathbf{I}_{n \times n}$. In other words, the assumption $\operatorname{trace}(A)=0$ is no restriction in generality because $A$ and $A-\frac{\operatorname{trace}(A)}{n} \cdot \mathbf{I}_{n \times n}$ have the same eigenvectors. The Kalman variety is identified with $\tilde{\mathcal{K}}_{d, n}=\tilde{\mathcal{K}}_{1, d, n}$ which has codimension $n-d$ and degree $\left(\begin{array}{c}n \\ d-1\end{array}\right)$ in $\mathbb{P}^{n^{2}-2}$. We shall derive the degree of $\tilde{\mathcal{K}}_{s, d, n}$, as promised in Theorem 4.6.

Let $G r\left(s, \mathbb{K}^{n}\right)$ be the $s(n-s)$-dimensional Grassmannian of $s$-planes in $\mathbb{K}^{n}$. Let $S$ be the universal bundle of rank $s$ and $Q$ the quotient bundle of rank $n-s$. They appear in the following exact sequence, with $\mathcal{O}$ the structure sheaf of $\operatorname{Gr}\left(s, \mathbb{K}^{n}\right)$ :

$$
0 \longrightarrow S \longrightarrow \mathcal{O} \otimes \mathbb{K}^{n} \longrightarrow Q \longrightarrow 0 .
$$

The fiber of $\operatorname{TGr}\left(s, \mathbb{K}^{n}\right)$ at $x=[V]$, where $V \subset \mathbb{K}^{n}$ is an $s$-dimensional subspace of $\mathbb{K}^{n}$, is isomorphic to $\operatorname{Hom}\left(V, \mathbb{K}^{n} / V\right)$. This means that $\operatorname{TGr}\left(s, \mathbb{K}^{n}\right)=\operatorname{Hom}(S, Q)$. Every traceless matrix $A \in$ ad $\mathbb{K}^{n}$ induces a section $s_{A} \in H^{0}\left(T G r\left(s, \mathbb{K}^{n}\right)\right)$ which corresponds to the composition $V \stackrel{i}{\longrightarrow} \mathbb{K}^{n} \stackrel{A}{\longrightarrow} \mathbb{K}^{n} \stackrel{\pi}{\longrightarrow} \mathbb{K}^{n} / V$ on the fiber of $[V]$. The section $s_{A}$ vanishes in $[V]$ if and only if $\pi(A(v))=0$ for all $v \in V$. This implies

Lemma 5.1. For $A \in a d \mathbb{K}^{n}$, the section $s_{A}$ vanishes in $V$ if and only if $A(V) \subseteq V$.

By the Borel-Weil Theorem, all sections $s$ of the tangent bundle $\operatorname{Tr} r\left(s, \mathbb{K}^{n}\right)$ have the form $s=s_{A}$ for some $A \in$ ad $\mathbb{K}^{n}$. Equivalently, $H^{0}\left(T G r\left(s, \mathbb{K}^{n}\right)\right)=$ ad $\mathbb{K}^{n}$. Since the generic traceless $n \times n$ matrix has $n$ distinct eigenvectors, a generic section of $\operatorname{TGr}\left(s, \mathbb{K}^{n}\right)$ vanishes in $\left(\begin{array}{l}n \\ s\end{array}\right)$ points, corresponding to the subspaces spanned by $s$ of the $n$ eigenvectors. We define $M^{s, n}$ to be the kernel of the evaluation map

$$
H^{0}\left(T G r\left(s, \mathbb{K}^{n}\right)\right) \otimes \mathcal{O} \longrightarrow T G r\left(s, \mathbb{K}^{n}\right) .
$$

Thus $M^{s, n}$ is a homogeneous bundle on $G r\left(s, \mathbb{K}^{n}\right)$ of rank $n(n-s)+\left(s^{2}-1\right)$. For $s=1$ we write $M^{1, n}=: M^{n}$. This is a homogeneous bundle of rank $n(n-1)$ on the projective space $\mathbb{P}^{n-1}$. For example, on $\operatorname{Gr}\left(1, \mathbb{K}^{2}\right)=\mathbb{P}^{1}$ we have $M^{2}=\mathcal{O}(-1)^{2}$.

The Kalman variety of a subspace $L$ in $\mathbb{K}^{n}$ (for $s=1$ ) and its singular strata (for $s \geq 2$ ) arise from the projectivization of the restriction of $M^{s, n}$ to $G r(s, L)$. Namely, we consider the product $X=\mathbb{P}\left(\operatorname{ad} \mathbb{K}^{n}\right) \times G r\left(s, \mathbb{K}^{n}\right)$ with its two projections

$$
\mathbb{P}\left(\operatorname{ad} \mathbb{K}^{n}\right) \swarrow^{X} \searrow^{q} G r\left(s, \mathbb{K}^{n}\right)
$$

Theorem 5.2. The desingularization of the variety $\tilde{\mathcal{K}}_{s, d, n}$ is isomorphic to the projective bundle $\mathbb{P}\left(M_{\mid G r(s, L)}^{s, n}\right)$, where $\operatorname{Gr}(s, L) \subset G r\left(s, \mathbb{K}^{n}\right)$ parametrizes planes in $L$. 
Proof. Generalizing (2.2), we have $\tilde{\mathcal{K}}_{d, s, n}(L)=p_{*}\left(\mathcal{J} \cap q^{*} G r(s, L)\right)$, where

$$
\mathcal{J}=\left\{(A, V) \in \mathbb{P}^{n^{2}-2} \times G r\left(s, \mathbb{K}^{n}\right) \mid A(V) \subseteq V\right\}
$$

is the incidence variety. By the same argument as in Corollary 4.2 , the subvariety $\mathcal{K} \mathcal{S}_{s, d, n}=\mathcal{J} \cap q^{*} \operatorname{Gr}(s, L)$ of $X$ coincides with the desingularization of $\tilde{\mathcal{K}}_{s, d, n}$. The fiber $q^{-1}(q(A, V))=q^{-1}(V)$ consists of all endomorphisms $A^{\prime}$ that have $V$ as an invariant subspace. By Lemma [5.1, this is the set of all sections of the tangent bundle which vanish at $V$, and hence it coincides with the fiber of $M^{s, n}$ at $V$.

Remark 5.3. After this paper had been written and refereed, we received Steven Sam's preprint [16, which uses Theorem 5.2 and the Weyman method to obtain the ideal sheaves of $\mathcal{K}_{2, n}$ and $\mathcal{K}_{3, n}$, among many other results. In particular, Sam proved our conjecture (Theorem 3.6 ) on the defining equations of $\mathcal{K}_{3, n}$.

The restriction $S_{\mid G r(s, L)}$ is the universal bundle on $\operatorname{Gr}(s, L)$. We denote this bundle again by $S$. The restriction $Q_{\mid G r(s, L)}$ splits as $Q_{L} \oplus \mathcal{O}^{n-d}$, where $Q_{L}$ is the quotient bundle of rank $d-s$ on $\operatorname{Gr}(s, L)$. The bundle appearing in Theorem 5.2 can then be described in the following way:

Proposition 5.4. We have $M_{\mid G r(s, L)}^{s, n}=M^{s, d} \oplus \mathcal{O}^{n(n-d)} \oplus\left[\left(Q_{L}\right)^{*}\right]^{n-d}$.

Proof. This is derived from the following commutative diagram on $\operatorname{Gr}(s, L)$ :

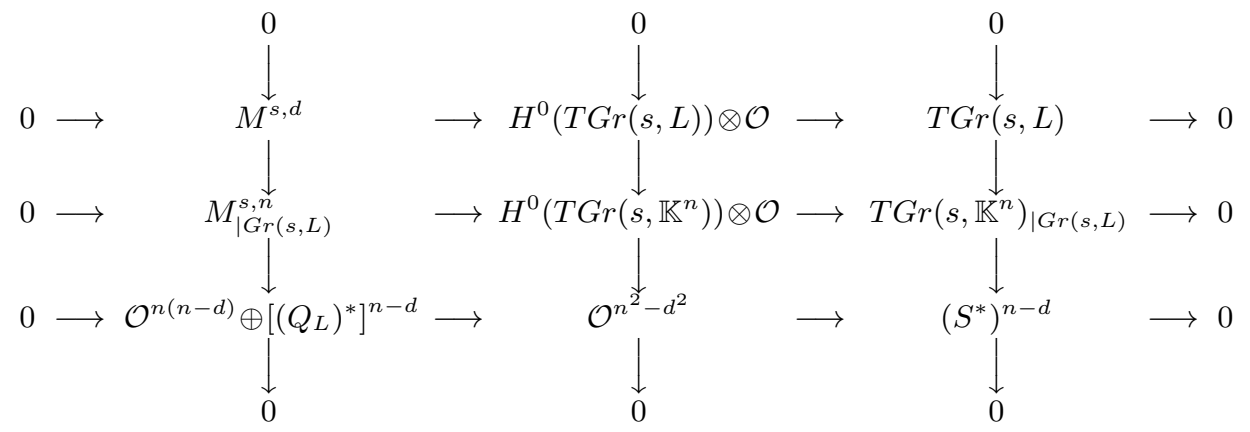

The second and third columns split, and hence also the first column splits.

The appearance of the trivial summand $\mathcal{O}^{n(n-d)}$ corresponds to the reduction (in the Introduction) from the Kalman matrix (1.1) to the small Kalman matrix (1.2). In the case $(s, d)=(1,2)$ of Section 3, the relevant Grassmannians are $\operatorname{Gr}(1, L)=$ $\mathbb{P}^{1} \subset \operatorname{Gr}\left(1, \mathbb{K}^{n}\right)=\mathbb{P}^{n-1}$, and Proposition 5.4 says that $M_{\mathbb{P} 1}^{n}=\mathcal{O}(-1)^{n} \oplus \mathcal{O}^{n(n-2)}$.

We recall (e.g. from [4]) that if a vector bundle $E$ of rank $r$ on a variety $X$ has a section vanishing on $Z$, and the codimension of $Z$ is equal to $r$, then the class of $[Z]$ in the degree $r$ component of the Chow ring $A^{r}(X)$ is computed by $[Z]=c_{r}(E)$. We shall apply this to the following vector bundle on the product variety $X$ in (5.2):

$$
E:=p^{*} \mathcal{O}(1) \otimes q^{*} \operatorname{TGr}\left(s, \mathbb{K}^{n}\right) .
$$

By Künneth's formula,

$$
H^{0}(X, E)=H^{0}\left(\mathbb{P}\left(\operatorname{ad} \mathbb{K}^{n}\right), \mathcal{O}(1)\right) \otimes H^{0}\left(T G r\left(s, \mathbb{K}^{n}\right)\right) \simeq \operatorname{End}\left(\operatorname{ad} \mathbb{K}^{n}\right) .
$$

Hence the identify map on ad $\mathbb{K}^{n}$ determines a canonical section $c \in H^{0}(E)$.

Proposition 5.5. The zero locus $Z(c)$ of c equals the incidence variety $\mathcal{J}$ in (5.3). 
Proof. We shall compute $c$ on the fiber of $E$ over $(A, V)$. The fiber is $\langle A\rangle \otimes$ $\operatorname{Hom}\left(V, \mathbb{K}^{n} / V\right)$ and the value of $c$ is $A \otimes\left(A_{\mid V}: V \rightarrow \mathbb{K}^{n} / V\right)$. So $c$ vanishes exactly at the pairs $(A, V)$ such that $V$ is $A$-invariant. This proves the assertion.

Since $Z(c)$ has codimension rk $E=s(n-s)$ in $X$ (e.g. by Theorem 4.4), the class of $Z(c)$ equals the top Chern class of $E$. In symbols, $[Z(c)]=c_{s(n-s)}(E)$.

Theorem 5.6. The degree of $\mathcal{K}_{s, d, n}$ equals $c_{s(d-s)}\left(\operatorname{TGr}\left(s, \mathbb{K}^{n}\right)\right) \cdot\left[c_{s}\left(S^{*}\right)\right]^{n-d}$.

Proof. The class of $G r(s, L) \subset G r\left(s, \mathbb{K}^{n}\right)$ is $\left[c_{s}\left(S^{*}\right)\right]^{n-d}$. The projection $p$ in (5.2) gives a birational map $Z(c) \cdot q^{*}\left[c_{s}\left(S^{*}\right)\right]^{n-d} \rightarrow \mathcal{K}_{s, d, n}$. The desired degree equals

$$
\operatorname{deg}\left(\mathcal{K}_{s, d, n}\right)=p^{*} c_{1}(\mathcal{O}(1))^{n^{2}-2-s(n-d)} \cdot c_{s(n-s)}(E) \cdot q^{*}\left(c_{s}\left(S^{*}\right)\right)^{n-d} .
$$

The Chern class of $E=p^{*} \mathcal{O}(1) \otimes q^{*} \operatorname{TGr}\left(s, \mathbb{K}^{n}\right)$ decomposes as

$$
c_{s(n-s)}(E)=\bigoplus_{i=0}^{s(n-s)} p^{*} c_{1}(\mathcal{O}(1))^{s(n-s)-i} \cdot q^{*} c_{i}\left(T G r\left(s, \mathbb{K}^{n}\right)\right) .
$$

Hence the quantity on the right of (5.4) can be written as

$$
\sum_{i=0}^{s(n-s)} p^{*} c_{1}(\mathcal{O}(1))^{n^{2}-2+s(d-s)-i} \cdot q^{*}\left[c_{i}\left(T G r\left(s, \mathbb{K}^{n}\right)\right) \cdot\left(c_{s}\left(S^{*}\right)\right)^{n-d}\right] .
$$

All summands are zero except for $i=s(d-s)$, and the result follows.

Proof of Theorem 4.6. The cohomology ring of the Grassmannian is identified with a ring of symmetric polynomials (see e.g. [5]), and under this identification we have

$$
c_{i}\left(S^{*}\right) \longleftrightarrow e_{i}=i \text {-th elementary symmetric function in } x_{1}, \ldots, x_{s} .
$$

From the sequence (5.1), tensored by $S^{*}$, we get the Chern polynomial

$$
p_{T G r\left(s, \mathbb{K}^{n}\right)}(t)=\frac{p_{S^{*}}(t)^{n}}{p_{S \otimes S^{*}}(t)}=\frac{\left(\sum_{i=0}^{s} c_{i}\left(S^{*}\right) t^{i}\right)^{n}}{\sum_{i=0}^{s} c_{i}\left(S \otimes S^{*}\right) t^{i}}=\frac{\prod_{i=1}^{s}\left(1+x_{i} t\right)^{n}}{\prod_{i, j=1}^{s}\left(1+\left(x_{i}-x_{j}\right) t\right)} .
$$

Theorem [5.6 and (5.5) imply the result. Note the analogy with (3) in [2, §16].

We end by describing a few special cases of Theorem 4.6. For $s=1$ we recover Proposition 1.2 for the original Kalman variety: $\operatorname{deg} \mathcal{K}_{1, d, n}=\operatorname{deg} c_{d-1} T \mathbb{P}^{n-1}=$ $\left(\begin{array}{c}n \\ d-1\end{array}\right)$. For $s=d-1$, the Grassmannian $\operatorname{Gr}\left(s, \mathbb{K}^{d}\right)$ is a projective space and the degree of $\mathcal{K}_{d-1, d, n}$ is the coefficient of $t^{d-1}$ in the rational function $(1+t)^{d} /(1-t)^{n-d}$. For $s=2$, the Chern polynomial in the above proof is the appropriate truncation of

$$
p_{T G r\left(2, \mathbb{K}^{n}\right)}(t)=\frac{\left(1+c_{1}\left(S^{*}\right) t+c_{2}\left(S^{*}\right) t^{2}\right)^{n}}{1-\left(c_{1}\left(S^{*}\right)^{2}-4 c_{2}\left(S^{*}\right)\right) t^{2}}=\frac{\left(1+x_{1} t\right)^{n}\left(1+x_{2} t\right)^{n}}{1-\left(x_{1}-x_{2}\right)^{2} t^{2}} .
$$

Christoph Koutschan from RISC-Linz kindly helped us by means of his software [11] for holonomic summation. Writing $(a)_{n}=\prod_{i=0}^{n-1}(a+i)$, he found the formula

$$
\operatorname{deg}\left(\mathcal{K}_{2, d, n}\right)=(-1)^{d} \frac{2^{2 d-3}}{(d-1) !} \sum_{k=0}^{d-2} \frac{(1 / 2-k)_{d-1}(n+1-k)_{k}(d+n-2 k)_{k}}{(2 k) !} .
$$

This expression is the degree of the singular locus of the Kalman variety $\mathcal{K}_{d, n}$. 
We note that the series expansion of (4.1) in terms of Schur polynomials can be computed using Stembridge's Maple package SF [18, or the more recent package Schubert2 in Macaulay2 [8]. For our computations we used the latter, by means of the following convenient function, which implements the formula in Theorem 5.6.

loadPackage "Schubert2"

$\mathrm{kal}=(\mathrm{s}, \mathrm{d}, \mathrm{n}) \rightarrow(\mathrm{G}=\mathrm{flagBundle}(\{\mathrm{s}, \mathrm{n}-\mathrm{s}\}) ;(\mathrm{S}, \mathrm{Q})=\mathrm{G}$. Bundles; $\left.\operatorname{integral}\left(\left((\operatorname{chern}(s, \operatorname{dual}(\mathrm{S})))^{\wedge}(\mathrm{n}-\mathrm{d})\right) *(\operatorname{chern}(\mathrm{s} *(\mathrm{~d}-\mathrm{s}),(\operatorname{dual}(\mathrm{S})) * * \mathrm{Q}))\right)\right)$

This little piece of code highlights the utility of vector bundle techniques as a practical tool for hands-on computations concerning problems in linear algebra.

\section{ACKNOWLEDGEMENT}

This project began in June 2010, after a visit by the second author to the Basque Center of Applied Mathematics in Bilbao, where Enrique Zuazua told him about the Kalman observability condition and its role for partial differential equations. Beauchard and Zuazua [1] had shown that the Kalman variety arises as the failure locus of the Shizuta-Kawashima condition for partially dissipative linear hyperbolic systems. One week after Bilbao, the second author visited Florence, where he discussed this topic with the first author, and this led to the present paper. We are grateful to Enrique for getting us started and for participating in our early e-mail discussions. We also thank Uwe Helmke and Raman Sanyal for helping us with references.

\section{REFERENCES}

[1] K. Beauchard and E. Zuazua: Large time asymptotics for partially hyperbolic systems, Arch. Rational Mech. Anal. 199 (2011) 177-227. MR2754341

[2] A. Borel and F. Hirzebruch: Characteristic classes and homogeneous spaces. I, American J. of Math. 80 (1958) 458-538. MR0102800 (21:1586)

[3] A. Compta, U. Helmke, M. Peña, and X. Puerta: Simultaneous versal deformations of endomorphisms and invariant subspaces, Linear Algebra Appl. 413 (2006) 303-318. MR2198936 (2006m:58060)

[4] W. Fulton: Intersection Theory, Springer, Berlin, 1984. MR732620 (85k:14004)

[5] W. Fulton: Young Tableaux, LMS Student Texts 35, Cambridge University Press, 1997. MR 1464693 (99f:05119)

[6] M. Hautus: Controllability and observability conditions of linear autonomous systems, Indagationes Mathematicae 31 (1969) 443-448. MR0250694(40:3926)

[7] U. Helmke and J. Trumpf: Conditioned invariant subspaces and the geometry of nilpotent matrices, in: New directions and applications in control theory, Lecture Notes in Control and Inform. Sci., 321, Springer, Berlin, 2005, 123-163. MR2180264 (2006h:93031)

[8] D. Grayson and M. Stillman: Macaulay 2, a software system for research in algebraic geometry, available at www.math.uiuc.edu/Macaulay2/.

[9] T. Kailath: Linear Systems, Prentice-Hall, Englewood Cliffs, 1980. MR569473 (82a:93001)

[10] R.E. Kalman: Contributions to the theory of optimal control, Bol. Soc. Mat. Mexicana (2) 5 (1960) 102-119. MR0127472 (23:B518)

[11] C. Koutschan: Advanced Applications of the Holonomic Systems Approach, PhD Thesis, RISC, Johannes Kepler University, Linz, Austria, 2009.

[12] E. Miller and B. Sturmfels: Combinatorial Commutative Algebra, Springer, New York, 2005. MR2110098(2006d:13001)

[13] N.I. Osetinskiŭ, O.O. Vasil'ev, and F.S. Vainshteřn: Geometric combinatorics of Kalman algebras, Differential Equations 42 (2006), no. 11, 1604-1611. MR2347083 (2008i:93048)

[14] T. Saaty: The Analytic Hierarchy Process: Planning, Priority Setting, Resource Allocation, McGraw-Hill, New York, 1980. MR773297 (86f:90009)

[15] T. Saaty and G. Hu: Ranking by eigenvector versus other methods in the analytic hierarchy process, Appl. Math. Lett. 11 (1998), no. 4, 121-125. MR.1631150(99b:90012) 
[16] S. Sam: Equations and syzygies of some Kalman varieties, Proc. Amer. Math. Soc. 140 (2012), 4153-4166.

[17] D. Shemesh: Common eigenvectors of two matrices, Linear Algebra Appl. 62 (1984) 11-18. MR761057 (85i:15016)

[18] J. Stembridge: A Maple package for symmetric functions, J. Symbolic Comput. 20 (1995), no. 5-6, 755-768. MR1395426 (97j:05061)

[19] N. Tran: Pairwise ranking: choice of method can produce arbitrarily different rank order, arXiv: 1103.1110 .

Department of Mathematics, University of Florence, viale Morgagni 67/A, 50134 FLORENCE, ITALy

E-mail address: ottavian@math.unifi.it

Department of Mathematics, University of California, Berkeley, California 94720

E-mail address: bernd@math.berkeley.edu 